\title{
Dipeptidyl peptidase-4 inhibitor sitagliptin prevents high glucose-induced apoptosis via activation of AMP-activated protein kinase in endothelial cells
}

\author{
CHAO WU ${ }^{1}$, SHUNYING HU ${ }^{2}$, NANPING WANG ${ }^{3,4}$ and JIANWEI TIAN ${ }^{5}$ \\ ${ }^{1}$ Postgraduate Department, Third Military Medical University, Chongqing 400038; ${ }^{2}$ Department of Cardiology, \\ Chinese PLA General Hospital, Beijing 100853; ${ }^{3}$ Institute of Cardiovascular Science, Peking University, Beijing 100191; \\ ${ }^{4}$ The Advanced Institute for Medical Sciences, Dalian Medical University, Dalian, Liaoning 116044; \\ ${ }^{5}$ Department of Cardiology, Air Force General Hospital, Beijing 100142, P.R. China
}

Received January 30, 2016; Accepted February 9, 2017

DOI: $10.3892 / \mathrm{mmr} .2017 .6501$

\begin{abstract}
Diabetes mellitus (DM), which is a chronic metabolic disorder, is the primary risk factor of life-threatening vascular complications. Endothelial apoptosis is important in the development of the initial vascular lesion preceding the diabetic disease. Sitagliptin is a dipeptidyl peptidase-4 (DPP-4) inhibitor and extensively used in the clinical treatment of DM. DPP-4 inhibitors have been demonstrated to be beneficial in the improvement of endothelial homeostasis, however the molecular mechanism by which they exhibit these effects remains to be elucidated. The effect of sitagliptin on endothelial apoptosis was examined in cultured human umbilical vein endothelial cells (HUVECs) incubated with high glucose (HG). The present study demonstrated that treatment of HUVECs with HG increased reactive oxygen species (ROS) production, stimulated mitochondrial depolarization and resulted in cell apoptosis. Pretreatment of HUVECs with sitagliptin significantly prevented HG-induced endothelial apoptosis. It was further demonstrated that sitagliptin effectively inhibited ROS generation and mitochondrial membrane potential collapse. Similarly, adenosine monophosphate-activated protein kinase (AMPK) activation by sitagliptin protected against HG-induced ROS production, mitochondrial membrane potential collapse
\end{abstract}

Correspondence to: Dr Jianwei Tian, Department of Cardiology, Air Force General Hospital, 30 Fucheng Road, Beijing 100142, P.R. China

E-mail: jianweitian2005@gmail.com

Abbreviations: DPP-4, dipeptidyl peptidase-4; ROS, reactive oxygen species; DM, diabetes mellitus; HG, high glucose; HUVECs, human umbilical vein endothelial cells; $\Delta \Psi \mathrm{m}$, mitochondrial membrane potential; AMPK, adenosine monophosphate-activated protein kinase

Key words: dipeptidyl peptidase-4 inhibitor, apoptosis, adenosine monophosphate-activated protein kinase, endothelial cell, reactive oxygen species and endothelial cell apoptosis, as detected via western blotting and flow cytometry analysis. The present study therefore revealed a novel mechanism of sitagliptin-mediated AMPK activation in preventing endothelial apoptosis and indicated the therapeutic potential of sitagliptin in vascular complications associated with endothelial apoptosis.

\section{Introduction}

Diabetes mellitus (DM) is an increasingly prevalent worldwide disease that is challenging human health and is currently of primary concern. As one of the major risk factors for cardiovascular diseases, type 2 diabetes contributes greatly to the occurrence of disabilities in later life and to mortality (1). Over $50 \%$ of mortality events resulting from type 2 diabetes are attributable to cardiovascular diseases (2), including stroke and myocardial infarction. Microvascular endothelial apoptosis is important in the development of the initial vessel lesions of vascular complications in DM (3).

Dipeptidyl peptidase-4 (DPP-4) inhibitors are of a class of oral hypoglycemic agents, which reduce blood glucose levels with a low risk of hypoglycemia and weight gain. DPP-4 is an enzyme expressed on blood vessels, myocardium and myeloid cells and is responsible for the inactivation of endogenous glucoregulatory peptides, termed incretins (4). Glucagon-like peptide 1 (GLP-1) and gastric inhibitory peptide are two well-studied incretins. GLP-1 prolongs gastric emptying, reduces appetite, inhibits glucagon release and stimulates insulin in a glucose-dependent manner. GLP-1 receptor (GLP-1R) agonists have been used in the treatment of patients with type 2 diabetes (5). Sitagliptin was the first clinically used DPP-4 inhibitor and was approved by the US Food and Drug Administration for the treatment of type 2 diabetes in 2006 (6). Recent studies in apolipoprotein E-deficient mice revealed that sitagliptin improved endothelial dysfunction, enhanced endothelial nitric oxide synthase (eNOS) phosphorylation (7) and reduced the atherosclerotic plaque area (8), suggesting that DPP-4 inhibitors may have further potential therapeutic effects beyond the incretin-dependent hypoglycemic action. DPP-4 inhibitors have been demonstrated to 
exhibit cardiovascular protective functions, however their effect on endothelial apoptosis and the underlying mechanism in diabetes remains to be fully elucidated.

Adenosine monophosphate-activated protein kinase (AMPK) is a cellular energy and stress sensor (9). In diabetes, AMPK has been observed to be dephosphorylated and inactive (10). A previous study revealed that AMPK activation significantly prevents the oxidative stress-induced apoptosis of human umbilical vein endothelial cells (HUVECs) (11). Various studies have additionally demonstrated that AMPK prevents apoptosis via inhibiting reactive oxygen species (ROS) generation by mitochondria (12) and nicotinamide adenine dinucleotide phosphate $[\mathrm{NAD}(\mathrm{P}) \mathrm{H}]$ oxidase (13). The molecular mechanism regarding how the DPP-4 inhibitor regulates endothelial homeostasis and the associated functional role of AMPK remains to be elucidated. The present study aimed to verify the mechanism by which the DPP-4 inhibitor sitagliptin protects against high glucose (HG)-induced vascular endothelial cell apoptosis and examine if AMPK is involved in this regulatory process.

\section{Materials and methods}

Reagents. Sitagliptin (phosphate) was provided by Cayman Chemical Company (Ann Arbor, MI, USA) and 5-aminoimidazole-4-carboxamide riboside (AICAR) was purchased from Beyotime Institute of Biotechnology (Haimen, China). Compound C was obtained from Sigma-Aldrich; Merck KGaA (Darmstadt, Germany). Monoclonal rabbit anti-phospho-AMPK $\alpha$ antibody (catalog no. 2535p) and anti-AMPK antibody (catalog no. 2603p) were obtained from Cell Signaling Technology, Inc. (Danvers, MA, USA). The following antibodies were also used: Monoclonal mouse anti- $\beta$-actin antibody (catalog no. sc-47778) and horseradish peroxidase-conjugated goat anti-rabbit/mouse secondary antibody (catalog no. sc-2004/sc-2005) from Santa Cruz Biotechnology, Inc. (Dallas, TX, USA).

Cell culture. HUVECs were isolated by collagenase digestion from fresh cord umbilical veins, as previously described (14). The flesh cord umbilical veins were obtained from normal cesarean section surgery. This was approved by Air Force General Hospital ethics committee with informed written consent. HUVECs between passages 3 and 6 were cultured in endothelial cell medium (ScienCell Research Laboratories, Inc., Carlsbad, CA, USA) containing basal medium, supplemented with $5 \%$ fetal bovine serum (Gibco; Thermo Fisher Scientific, Inc., Waltham, MA, USA) and 1\% endothelial cell growth supplement with antibiotics $(100 \mathrm{U} / \mathrm{ml}$ penicillin $\mathrm{G}$ and $100 \mu \mathrm{g} / \mathrm{ml}$ streptomycin sulfate). This was conducted in a humidified atmosphere containing $5 \% \mathrm{CO}_{2}$, at $37^{\circ} \mathrm{C}$.

Western blotting. To determine the effect of sitagliptin on AMPK activation, the HUVECs were treated with $1 \mu \mathrm{M}$ sitagliptin for $0.5,1,2$ and $4 \mathrm{~h}$ or $100 \mu \mathrm{M}$ AMPK activator AICAR, for $0.5 \mathrm{~h}$. To detect the inhibitory effect of the AMPK inhibitor compound $\mathrm{C}$ on sitagliptin-induced AMPK $\alpha$ phosphorylation, HUVECs were incubated with $1 \mu \mathrm{M}$ sitagliptin, $10 \mu \mathrm{M}$ compound $\mathrm{C}$ or $1 \mu \mathrm{M}$ sitagliptin plus $10 \mu \mathrm{M}$ compound $\mathrm{C}$ for $2 \mathrm{~h}$. The cytoplasmic protein of cells was extracted with ice-cold hypotonic lysis buffer
[50 mM Tris-HCl, pH 7.5, 15 mM EGTA, 0.1\% (vol/vol) Triton $\mathrm{X}-100,100 \mathrm{mM} \mathrm{NaCl}$ and complete protease inhibitor cocktail] as previously described (15). Cell lysates were first snap frozen in liquid nitrogen and then centrifuged at $12,000 \mathrm{x}$ g at $4^{\circ} \mathrm{C}$ for $20 \mathrm{~min}$, for collection of the supernatant. Protein concentration was measured using the BCA method. Equal amounts of protein (10 $\mu \mathrm{g}$ per sample) were separated on $10 \%$ sodium dodecyl sulfate-polyacrylamide gels electrophoresis and blotted onto polyvinylidene difluoride membranes. Following incubation with no fat milk at $25^{\circ} \mathrm{C}$ for $20 \mathrm{~min}$, the membranes were reacted with anti-phospho-AMPK $\alpha$ antibody $(1: 1,000)$ and anti-AMPK $\alpha$ antibody $(1: 1,000)$ at $4^{\circ} \mathrm{C}$ overnight, then reacted with appropriate horseradish peroxidase-conjugated secondary antibodies $(1: 3,000)$ for $2 \mathrm{~h}$ at $25^{\circ} \mathrm{C}$. Proteins were visualized with an enhanced chemiluminescence kit, as previously described (16). Densitometry analysis was performed for three independent experiments using the Image J Gel Analysis tool (National Institutes of Health, Bethesda, MD, USA).

Measurement of endothelial apoptosis. HUVECs $\left(1 \times 10^{5}\right)$ were incubated with $\mathrm{HG}(33 \mathrm{mM})$ in the presence of $1 \mu \mathrm{M}$ sitagliptin, $100 \mu \mathrm{M}$ AICAR or $1 \mu \mathrm{M}$ sitagliptin plus $10 \mu \mathrm{M}$ AMPK inhibitor compound $\mathrm{C}$ for $48 \mathrm{~h}$. Induction of apoptosis in the treated groups was assessed by Annexin V-fluorescein isothiocyanate (FITC)/propidium iodide (PI) double staining detection kit (Nanjing KeyGen Biotech Co., Ltd., Nanjing, China), according to the manufacturer's protocol. Briefly, cells were incubated with $33 \mathrm{mM}$ D-glucose in the presence of the aforementioned agents for $48 \mathrm{~h}$ and gently digested with $1 \mathrm{ml} \mathrm{0.25 \%} \mathrm{trypsin} \mathrm{(Thermo} \mathrm{Fisher} \mathrm{Scientific,} \mathrm{Inc.)} \mathrm{for} 2 \mathrm{~min}$. The trypsinized cells were washed once with endothelial cell medium containing $5 \%$ fetal bovine serum prior to collection by centrifugation at $1,000 \mathrm{x} \mathrm{g}$ and room temperature for $20 \mathrm{~min}$. Cells were resuspended in $500 \mu \mathrm{l}$ of $1 \mathrm{X}$ binding buffer, followed by incubation with $5 \mu$ l of Annexin V-FITC and $5 \mu \mathrm{l}$ of $\mathrm{PI}(50 \mu \mathrm{g} / \mathrm{ml})$ for $10 \mathrm{~min}$ in the dark. Binding buffer, Annexin V-FITC and PI are components of the detection kit. All procedures subsequent to cell incubation were performed at room temperature. Stained cells were monitored by flow cytometry (BD FACSCalibur; BD Biosciences, San Jose, CA, USA) and analyzed with BD FACSDiva ${ }^{\mathrm{TM}}$ software (version 6.0; BD Biosciences).

Measurement of ROS generation. A ROS-specific fluorescent probe, 2',7'-dichlorodihydrofluorescein diacetate $\left(\mathrm{H}_{2} \mathrm{DCFDA}\right.$; Molecular Probes; Thermo Fisher Scientific, Inc.) was used for the measurement of cytosolic ROS production. HUVECs were incubated with $33 \mathrm{mM}$ D-glucose in the presence of $1 \mu \mathrm{M}$ sitagliptin, $100 \mu \mathrm{M}$ AICAR or $1 \mu \mathrm{M}$ sitagliptin plus $10 \mu \mathrm{M}$ compound $\mathrm{C}$ for $48 \mathrm{~h}$, then cells were stained with $10 \mu \mathrm{M} \mathrm{H}_{2}$ DCFDA fluorescent probe in serum-free endothelial cell medium at $37^{\circ} \mathrm{C}$ for $30 \mathrm{~min}$. The labeled cells were then washed twice with serum-free endothelial cell medium and the levels of ROS were immediately analyzed by flow cytometry (BD FACSCalibur; BD Biosciences).

Mitochondrial membrane potential $(\Delta \Psi m)$ assay. To measure $\triangle \Psi \mathrm{m}$, HUVECs were treated with $1 \mu \mathrm{M}$ sitagliptin, $100 \mu \mathrm{M}$ AICAR or $1 \mu \mathrm{M}$ sitagliptin plus $10 \mu \mathrm{M}$ compound $\mathrm{C}$ prior to exposure to $33 \mathrm{mM}$ D-glucose for $48 \mathrm{~h}$. Following incubation, 

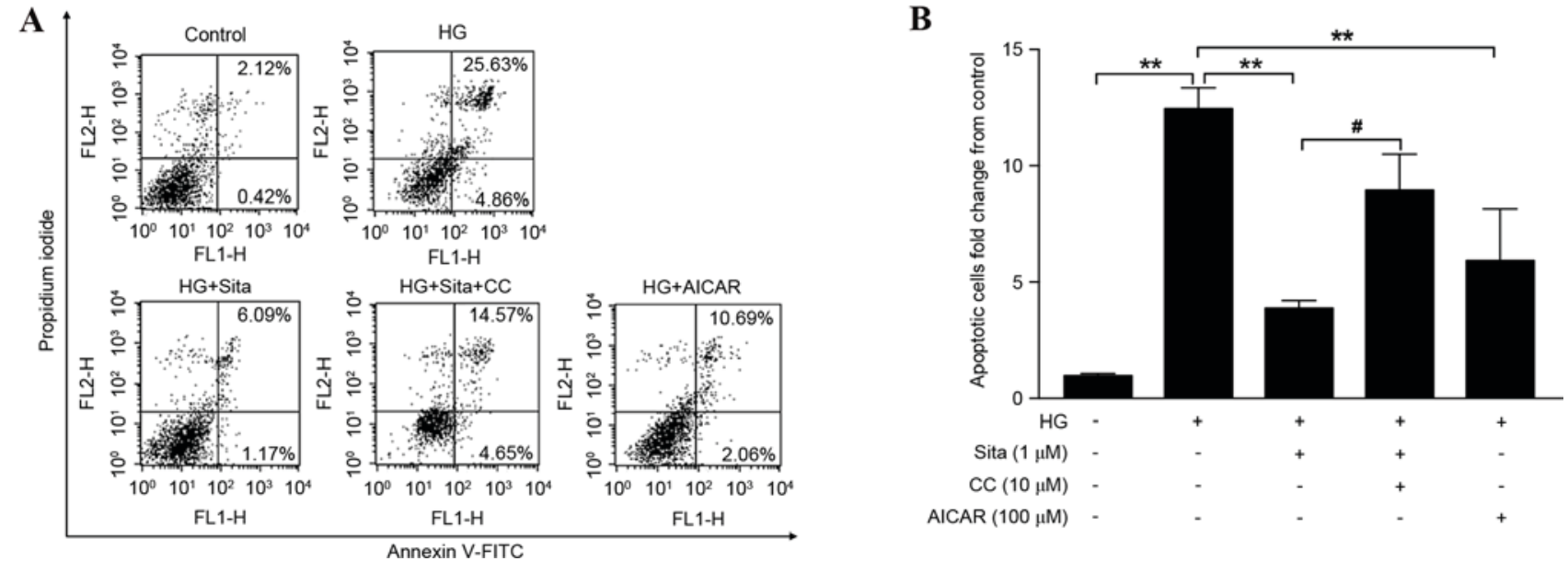

Figure 1. Sitagliptin prevents HG-induced apoptosis in vascular endothelial cells. (A) The apoptosis rate of endothelial cells in the five groups was assessed by Annexin V-FITC/PI staining and monitored with flow cytometry. The lower right quadrant: Annexin V-FITC-positive/PI-negative cells, representing early apoptotic cells. The upper right quadrant: Annexin V-FITC-positive/PI-positive cells, indicating late apoptotic cells. The values represent the percentage of the total cells in the two quadrants. (B) Quantification of apoptotic cell rate compared with control group. HG-induced cell apoptosis was prevented by sitagliptin or AMPK activator, AICAR. Compound C, an AMPK inhibitor reversed inhibition of cell apoptosis by sitagliptin. Data are expressed as the mean \pm standard error of the mean from three independent experiments. ${ }^{* *} \mathrm{P}<0.01$ vs. HG group, ${ }^{\#} \mathrm{P}<0.05$. Sita, sitagliptin; CC, compound C; HG, high glucose; AICAR, 5 -aminoimidazole-4-carboxamide riboside; FITC, fluorescein isothiocyanate; PI, propidium iodide.

cells were collected and stained with $2 \mu \mathrm{M} \Delta \Psi \mathrm{m}$-specific fluorescent dye JC-1 (Molecular Probes; Thermo Fisher Scientific, Inc.) at $37^{\circ} \mathrm{C}$ in an atmosphere containing $5 \% \mathrm{CO}_{2}$, for $20 \mathrm{~min}$. Flow cytometry (BD FACSCalibur; BD Biosciences) was used to detect $\Delta \Psi \mathrm{m}$ for each treatment group. JC-1 accumulates in mitochondria in a potential-dependent manner. In normal mitochondria with high $\Delta \Psi \mathrm{m}$, JC-1 aggregates with red fluorescence. In apoptotic cells with injured mitochondria membrane, JC-1 alters to monomers, and emits green fluorescence. $\Delta \Psi \mathrm{m}$ is determined by red/green fluorescence intensity ratio.

Statistical analysis. Data are expressed as the mean \pm standard error of the mean. One-way analysis of variance was used to determine differences among the mean values of treatments. SPSS software, version 20.0 (IBM SPSS, Armonk, NY, USA) was used for the statistical data analysis. $\mathrm{P}<0.05$ was considered to indicate a statistically significant difference.

\section{Results}

Sitagliptin prevents $H G$-induced endothelial apoptosis. The present study examined the effect of sitagliptin on HUVECs incubated with HG. Cell apoptosis of the pretreated groups was measured by Annexin V-FITC/PI double staining and monitored by flow cytometry (Fig. 1A). It was observed that HG significantly increased cell apoptosis, and this HG-induced endothelial cell apoptosis was prevented by sitagliptin or the AMPK activator, AICAR. However, compound C, an AMPK inhibitor, reversed the inhibition of apoptosis by sitagliptin (Fig. 1B). This therefore indicated that AMPK is important in the regulatory action of sitagliptin.

Sitagliptin activates AMPKa phosphorylation in vascular endothelial cells. As AMPK was observed to be involved in sitagliptin-mediated prevention of endothelial cell apoptosis, the present study aimed to determine the effect of sitagliptin on AMPK activity. HUVECs were incubated with $1 \mu \mathrm{M}$ sitagliptin at different times ranging from 0.5-4 $\mathrm{h}$. Phosphorylation of AMPK $\alpha$ (p-AMPK $\alpha$ ) was determined by western blotting (Fig. 2A). Sitagliptin stimulated AMPK $\alpha$ $\left(\mathrm{Thr}^{172}\right)$ phosphorylation from $2 \mathrm{~h}$, and this phosphorylation activity prevailed until $4 \mathrm{~h}$. AICAR enhanced AMPK phosphorylation in endothelial cells in a similar manner to sitagliptin, following incubation with the cells for $0.5 \mathrm{~h}$ (Fig. 2B). The effect of compound $\mathrm{C}$ on sitagliptin-induced AMPK $\alpha$ phosphorylation was additionally examined (Fig. 2C). As presented in (Fig. 2D), sitagliptin-stimulated AMPK $\alpha$ activation was significantly inhibited by compound C. These findings suggested that sitagliptin induces AMPK $\alpha$ phosphorylation.

Sitagliptin decreases high glucose-induced ROS generation. In vascular endothelial cells, the hyperglycemia load increases generation of ROS (17), which subsequently contributes to cell apoptosis. To observe the effect of sitagliptin pretreatment on HG-induced cytosolic ROS generation, cytosolic ROS levels were detected via flow cytometry (Fig. 3A). It was observed that high glucose significantly increased ROS production, however this was suppressed with pretreatment with $1 \mu \mathrm{M}$ sitagliptin. In addition, AICAR effectively inhibited generation of ROS, whereas compound $\mathrm{C}$ diminished the inhibitory effect of sitagliptin (Fig. 3B). These data suggested that sitagliptin inhibits cytosolic ROS via AMPK activation. The effect of sitagliptin on ROS-mediated mitochondrial dysfunction, under conditions of hyperglycemia were then examined.

Sitagliptin restores the loss of $\Delta \Psi \mathrm{m}$. ROS-mediated $\Delta \Psi \mathrm{m}$ collapse was previously demonstrated to initiate mitochondrial-dependent apoptosis in DM (18). The present study proceeded to characterize HG-induced $\Delta \Psi \mathrm{m}$ alterations and examine if sitagliptin protects against $\Delta \Psi \mathrm{m}$ collapse. JC-1 staining detection by flow cytometry was performed. 


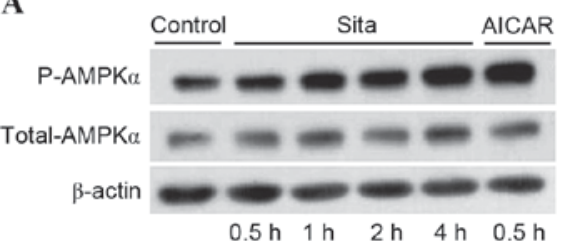

C

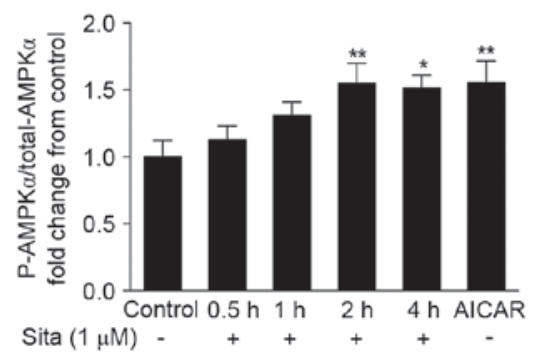

B

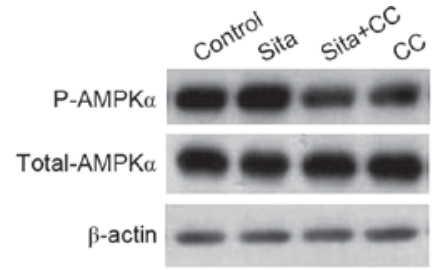

D

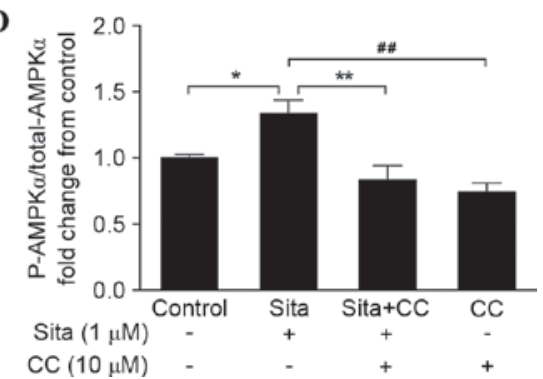

Figure 2. Sitagliptin activates AMPK $\alpha$ phosphorylation. (A) Phosphorylation of AMPK $\alpha$ was determined by western blotting. AMPK $\alpha$ phosphorylation was enhanced from 2-4 h. AICAR additionally activated AMPK $\alpha$ phosphorylation. (B) Compound C inhibited sitagliptin-induced AMPK $\alpha$ activation. The results were quantified and expressed as AMPK $\alpha$ phosphorylation normalized to total AMPK $\alpha$ following (C) sitagliptin plus AICAR treatment ("P $<0.05$, ${ }^{* *} \mathrm{P}<0.01$ vs. control), and (D) sitagliptin plus compound C, in bar graphs. Data are presented as the mean \pm standard error of the mean of three independent experiments ( $\left(\mathrm{P}<0.05,{ }^{* *} \mathrm{P}<0.01,{ }^{\# \#} \mathrm{P}<0.01\right)$. Sita, sitagliptin; CC, compound C; AICAR, 5-aminoimidazole-4-carboxamide riboside; AMPK, adenosine monophosphate-activated protein kinase; $\mathrm{P}$, phosphorylated.
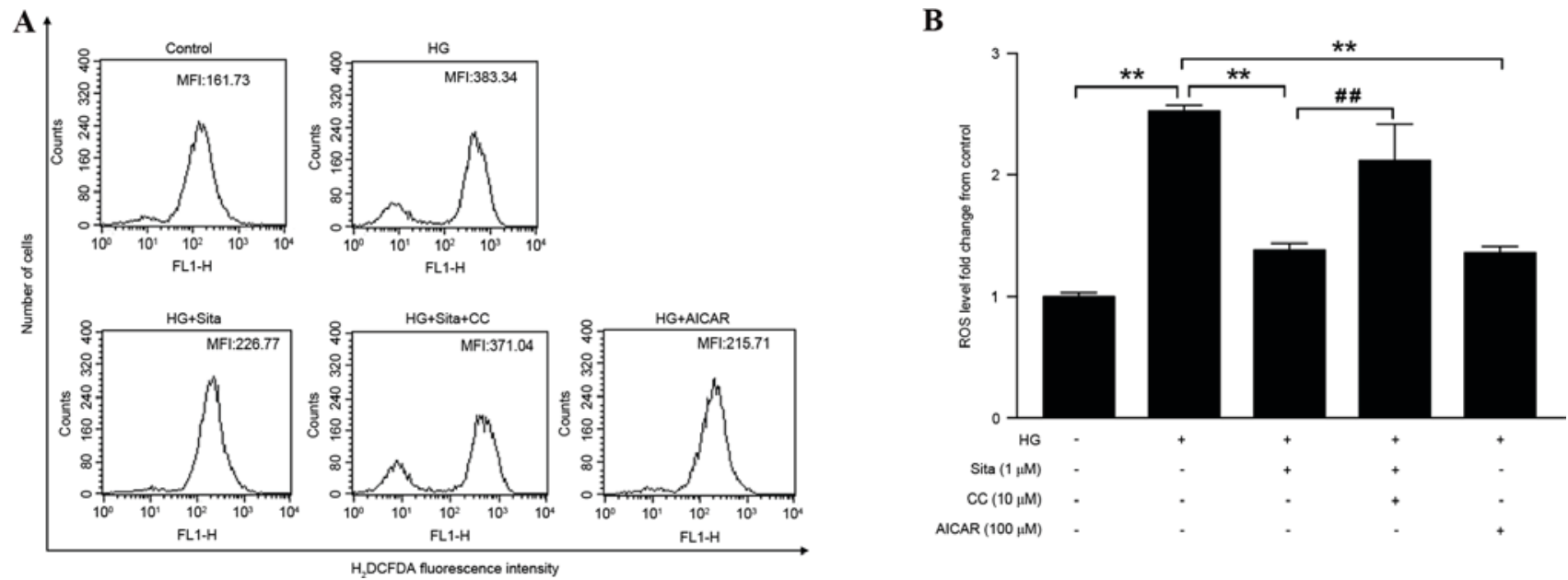

Figure 3. Sitagliptin decreases HG-induced cytosolic ROS generation. (A) The MFI of the cells was monitored using flow cytometry. (B) ROS level was quantified as MFI of each group compared with control group. Sitagliptin decreased HG-induced ROS generation, which was blocked by compound C. AICAR similarly inhibited hyperglycemia-induced intracellular ROS. Data are presented as the mean \pm standard error of the mean of three independent experiments. ${ }^{* *} \mathrm{P}<0.01,{ }^{\# \#} \mathrm{P}<0.01$. Sita, sitagliptin; $\mathrm{CC}$, compound $\mathrm{C} ; \mathrm{HG}$, high glucose; ROS, reactive oxygen species; AICAR, 5-aminoimidazole-4-carboxamide riboside; MFI, mean fluorescence intensity; $\mathrm{H}_{2}$ DCFDA, 2',7'-dichlorodihydrofluorescein diacetate.

Mitochondrial depolarization is determined by a decrease in aggregate/monomer fluorescence ratio (Fig. 4A). In a similar manner to that exhibited by AICAR, $1 \mu \mathrm{M}$ sitagliptin restored HG-induced $\Delta \Psi \mathrm{m}$ collapse, and this effect was blocked by compound C (Fig. 4B). These results suggested that AMPK is important in the regulatory actions of sitagliptin in $\mathrm{HG}$-induced endothelial apoptosis.

\section{Discussion}

The present study demonstrated that the DPP-4 inhibitor, sitagliptin, functions as a regulator of endothelial cell apoptosis. In HUVECs, sitagliptin effectively prevented HG-induced apoptosis. The underlying mechanisms may involve inhibition of ROS and the downstream $\Delta \Psi \mathrm{m}$ collapsing pathway, induced via AMPK $\alpha$ activation, as summarized in Fig. 5. Overall, the present study has reported novel findings, suggesting DPP-4 inhibitor-mediated AMPK activation as a therapeutic target for vascular endothelial apoptosis.

Diabetes is a major risk factor for cardiovascular disease. The risk of the development of cardiovascular complications in diabetes suggests a need for further therapeutic treatments, which may modulate disease-specific mechanisms, including endothelial apoptosis. DPP-4 inhibitors, including sitagliptin, alogliptin and vildagliptin are safe, well-tolerated hypoglycemic agents that have exhibited beneficial therapeutic effects in diabetes. Previous studies have demonstrated that DPP-4 inhibitors have substantial implications in the 

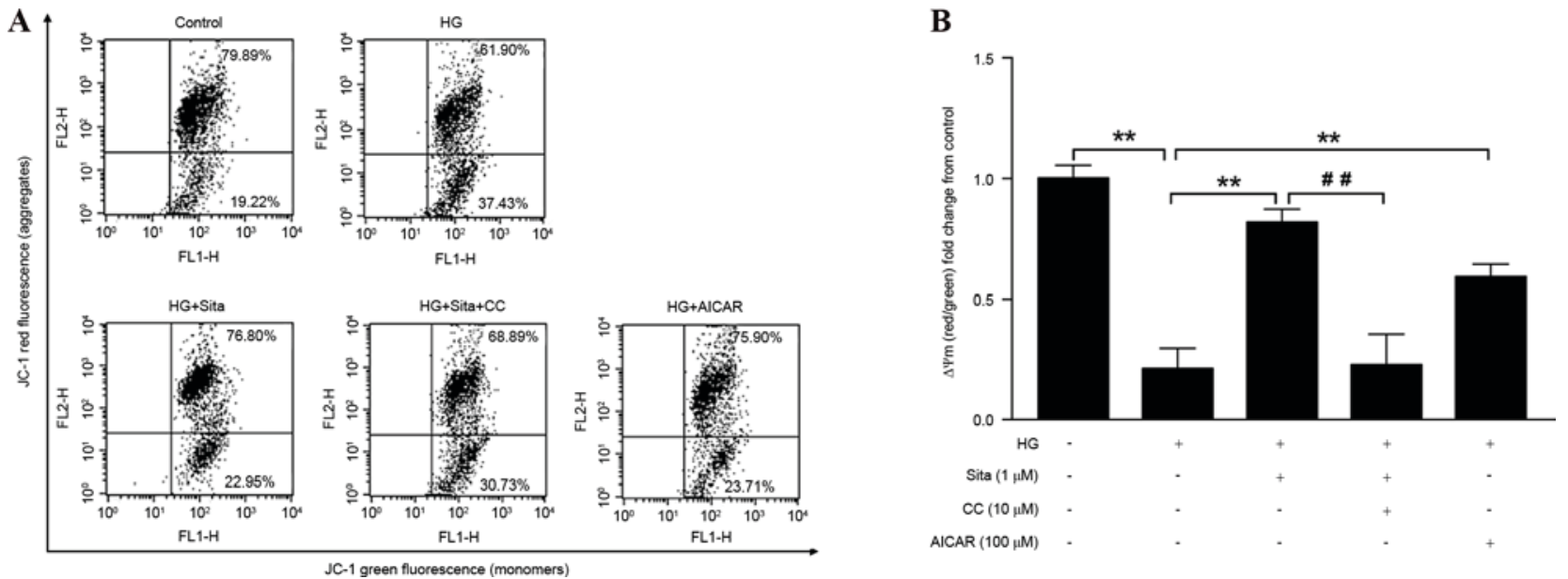

Figure 4. Sitagliptin restored $\Delta \Psi \mathrm{m}$ collapse in endothelial cells. (A) Representative flow cytometry graph. The mitochondria membrane depolarization is indicated by a fluorescence emission shift from red to green. Values are refer to the percentage of total cells in each quadrant. Data from three independent experiments are expressed as the mean \pm standard error of the mean. (B) Representation of red/green fluorescence intensity ratio. Sitagliptin restored HG-induced $\Delta \Psi \mathrm{m}$ collapse, in a similar manner to that exhibited by AICAR. Compound $\mathrm{C}$ reversed prevention of $\Delta \Psi \mathrm{m}$ collapse by sitagliptin. ${ }^{* *} \mathrm{P}<0.01$, ${ }^{\# \#} \mathrm{P}<0.01 . \Delta \Psi \mathrm{m}$, mitochondrial membrane potential; Sita, sitagliptin; CC, compound C; HG, high glucose; AICAR, 5-aminoimidazole-4-carboxamide riboside.

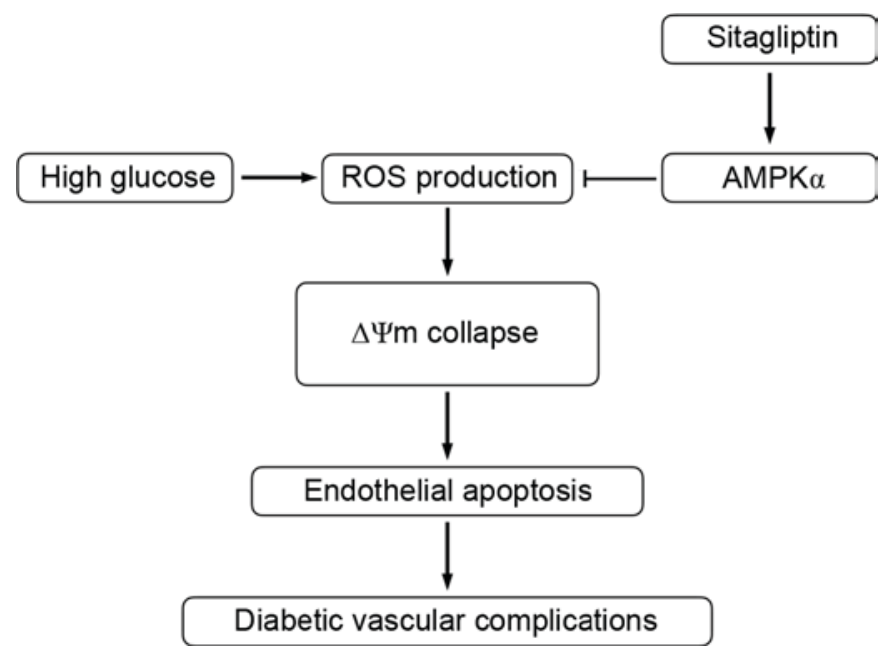

Figure 5. The proposed action pathway for sitagliptin in the prevention of diabetic vascular complications. In endothelial cells, high glucose exposure induces ROS production and the associated downstream $\Delta \Psi \mathrm{m}$ collapse, leading to mitochondria-dependent apoptosis. Sitagliptin inhibits high glucose-induced ROS production by activating AMPKs and effectively inhibits $\Delta \Psi \mathrm{m}$ collapse. Sitagliptin results in cell survival against high glucose exposure, and thereby prevents diabetic vascular complications under conditions of hyperglycemia. AMPK, adenosine monophosphate-activated protein kinase; ROS, reactive oxygen species.

cardiovascular system. Alogliptin relaxes reconstructed aortic segments (19), and incubation of HUVECs with vildagliptin has been demonstrated to result in phosphorylation of eNOS and serine/threonine kinase 1, increasing nitric oxide synthesis (20). Various animal model studies have demonstrated beneficial effects of DPP-4 inhibitors in improving blood pressure and endothelial dysfunction $(7,21)$. The present study revealed the novel mechanism of DPP-4 inhibitor-mediated apoptosis prevention. The results demonstrated that sitagliptin protected against HG-induced apoptosis in HUVECs, an effect additionally exhibited by the AMPK activator AICAR. Compound C, an AMPK inhibitor, diminished the inhibitory effect of the sitagliptin pretreatment. The potential link between sitagliptin and AMPK was then determined. The data demonstrated that AMPK $\alpha$ phosphorylation was activated by sitagliptin and compound $\mathrm{C}$ inhibited sitagliptin-induced AMPK $\alpha$ activation. Therefore, it was demonstrated that AMPK activation is important in sitagliptin-mediated protection against HG-induced vascular endothelial apoptosis.

AMPK is composed of catalytic $\alpha$-subunit and regulatory $\beta$ - and $\gamma$-subunits (9). The activation of AMPK occurs via $\alpha$-subunit phosphorylation at $\mathrm{Thr}^{172}(22)$. AMPK is an important regulator of metabolic homeostasis, and is considered a therapeutic target for the prevention of diabetic complications (23). Various reports have demonstrated that the AMPK signaling pathway exhibits a protective effect against endothelial dysfunction (24) and prevents apoptosis of HUVECs (11) consistent with the findings of the present study. A previous study reported that AMPK $\alpha$ acts as a physiological suppressor of NAD(P)H oxidase and ROS generation in endothelial cells (12), whereas a further study conversely indicated that AMPK is activated by ROS (25). Hyperglycemia-induced intracellular ROS production and associated downstream mitochondrial fission, stimulated $\Delta \Psi \mathrm{m}$ collapse, which resulted in mitochondrial-dependent apoptosis (26). The present study observed a significant increase in cytosolic ROS generation and $\Delta \Psi \mathrm{m}$ collapse upon incubation with HG in HUVECs. The ROS production and $\Delta \Psi \mathrm{m}$ collapse were suppressed by pretreatment with sitagliptin or AICAR. Compound C reversed the effect of sitagliptin. Therefore, there may be a negative feedback loop between AMPK and ROS, in which ROS generation potentiates AMPK activation, resulting in a further inhibition in intracellular ROS production.

Sitagliptin was demonstrated to prevent endothelial apoptosis via AMPK $\alpha$ activation, therefore it is necessary to elucidate the mechanism by which sitagliptin activates AMPK $\alpha$. The $\mathrm{Ca}^{2+} /$ calmodulin-activated protein kinase kinases (CaMKK), particularly CaMKK $\beta$ (27) and the liver kinase B1 (LKB1)-STRAD-MO25 complex (28) are major upstream kinases of AMPK in mammals, and the upstream 
kinase LKB1 is important for the activation of AMPK by AICAR (29). Sitagliptin mimicked the preventive effect of AICAR on HG-induced ROS production, $\Delta \Psi \mathrm{m}$ collapse, and endothelial cell apoptosis, indicating that sitagliptin-mediated AMPK HG-induced AMPK $\alpha$ activation may involve LKB1. It was additionally reported that sitagliptin treatment improved endothelial function in vivo via sequential activation of the LKB1/AMPKa/eNOS axis (30).

In conclusion, the results of the present study indicated that the DPP-4 inhibitor sitagliptin effectively prevented HG-induced cytosolic ROS generation, $\Delta \Psi \mathrm{m}$ collapse and apoptosis via activation of AMPK $\alpha$ in endothelial cells. These results suggested sitagliptin may act as a potential novel therapeutic agent to treat vascular complications in diabetes.

\section{Acknowledgements}

The present study was supported by The National Natural Science Foundation of China (grant no. 81070209). The authors would like to thank Ms. Guohua Ma (Department of Cardiology, Beijing Tian Tan Hospital, Beijing, China) for providing HUVECs, Dr Zhipeng Wang (Institute of Cardiovascular Science, Peking University, Beijing, China) for helpful discussions and Dr Yahan Liu (Institute of Cardiovascular Science, Peking University) for technical assistance.

\section{References}

1. Mazzone T, Chait A and Plutzky J: Cardiovascular disease risk in type 2 diabetes mellitus: Insights from mechanistic studies. Lancet 371: 1800-1809, 2008.

2. Zhong J, Maiseyeu A, Davis SN and Rajagopalan S: DPP4 in cardiometabolic disease: Recent insights from the laboratory and clinical trials of DPP4 inhibition. Circ Res 116: 1491-1504, 2015.

3. Libby P: Inflammation in atherosclerosis. Nature 420: 868-874, 2002.

4. Drucker DJ and Nauck MA: The incretin system: Glucagon-like peptide-1 receptor agonists and dipeptidyl peptidase-4 inhibitors in type 2 diabetes. Lancet 368: 1696-1705, 2006.

5. Ussher JR and Drucker DJ: Cardiovascular biology of the incretin system. Endocr Rev 33: 187-215, 2012.

6. Drucker D, Easley C and Kirkpatrick P: Sitagliptin. Nat Rev Drug Discov 6: 109-110, 2007.

7. Matsubara J, Sugiyama S, Sugamura K, Nakamura T, Fujiwara Y, Akiyama E, Kurokawa H, Nozaki T, Ohba K, Konishi M, et al: A dipeptidyl peptidase-4 inhibitor, des-fluoro-sitagliptin, improves endothelial function and reduces atherosclerotic lesion formation in apolipoprotein E-deficient mice. J Am Coll Cardiol 59: 265-276, 2012

8. Zeng Y, Li C, Guan M, Zheng Z, Li J, Xu W, Wang L, He F and Xue Y: The DPP-4 inhibitor sitagliptin attenuates the progress of atherosclerosis in apolipoprotein-E-knockout mice via AMPKand MAPK-dependent mechanisms. Cardiovasc Diabetol 13: 32, 2014.

9. Hardie DG, Ross FA and Hawley SA: AMPK: A nutrient and energy sensor that maintains energy homeostasis. Nat Rev Mol Cell Biol 13: 251-262, 2012.

10. Eid AA, Ford BM, Block K, Kasinath BS, Gorin Y, Ghosh-Choudhury G, Barnes JL and Abboud HE: AMP-activated protein kinase (AMPK) negatively regulates Nox4-dependent activation of p53 and epithelial cell apoptosis in diabetes. J Biol Chem 285: 37503-37512, 2010.

11. Ido Y, Carling D and Ruderman N: Hyperglycemia-induced apoptosis in human umbilical vein endothelial cells: Inhibition by the AMP-activated protein kinase activation. Diabetes 51: 159-167, 2002.
12. Wang S, Zhang M, Liang B, Xu J, Xie Z, Liu C, Viollet B, Yan D and Zou MH: AMPKalpha2 deletion causes aberrant expression and activation of NAD(P)H oxidase and consequent endothelial dysfunction in vivo: Role of $26 \mathrm{~S}$ proteasomes. Circ Res 106: $1117-1128,2010$.

13. Colombo SL and Moncada S: AMPKalpha1 regulates the antioxidant status of vascular endothelial cells. Biochem J 421: 163-169, 2009.

14. Pan B, Yu B, Ren H, Willard B, Pan L, Zu L, Shen X, Ma Y, Li X, Niu C, et al: High-density lipoprotein nitration and chlorination catalyzed by myeloperoxidase impair its effect of promoting endothelial repair. Free Radic Biol Med 60: 272-281, 2013.

15. Mao G, Liu Y, Fang X, Liu Y, Fang L, Lin L, Liu X and Wang N: Tumor-derived microRNA-494 promotes angiogenesis in non-small cell lung cancer. Angiogenesis 18: 373-382, 2015.

16. Yin R, Fang L, Li Y, Xue P, Li Y, Guan Y, Chang Y, Chen C and Wang N: Pro-inflammatory Macrophages suppress PPAR $\gamma$ activity in Adipocytes via S-nitrosylation. Free Radic Biol Med 89: 895-905, 2015.

17. Paneni F, Mocharla P, Akhmedov A, Costantino S, Osto E, Volpe M, Lüscher TF and Cosentino F: Gene silencing of the mitochondrial adaptor p66(Shc) suppresses vascular hyperglycemic memory in diabetes. Circ Res 111: 278-289, 2012.

18. Yee C, Yang W and Hekimi S: The intrinsic apoptosis pathway mediates the pro-longevity response to mitochondrial ROS in C. Elegans. Cell 157: 897-909, 2014.

19. Shah Z, Pineda C, Kampfrath T, Maiseyeu A, Ying Z, Racoma I, Deiuliis J, Xu X, Sun Q, Moffatt-Bruce S, et al: Acute DPP-4 inhibition modulates vascular tone through GLP-1 independent pathways. Vascul Pharmacol 55: 2-9, 2011.

20. Ishii M, Shibata R, Kondo K, Kambara T, Shimizu Y, Tanigawa T, Bando YK, Nishimura M, Ouchi N and Murohara T: Vildagliptin stimulates endothelial cell network formation and ischemia-induced revascularization via an endothelial nitric-oxide synthase-dependent mechanism. J Biol Chem 289: 27235-27245, 2014.

21. Aroor AR, Sowers JR, Bender SB, Nistala R, Garro M, Mugerfeld I, Hayden MR, Johnson MS, Salam M, Whaley-Connell A and Demarco VG: Dipeptidylpeptidase inhibition is associated with improvement in blood pressure and diastolic function in insulin-resistant male Zucker obese rats. Endocrinology 154: 2501-2513, 2013.

22. Oakhill JS, Steel R, Chen ZP, Scott JW, Ling N, Tam S and Kemp BE: AMPK is a direct adenylate charge-regulated protein kinase. Science 332: 1433-1435, 2011.

23. Viollet B, Lantier L, Devin-Leclerc J, Hebrard S, Amouyal C, Mounier R, Foretz M and Andreelli F: Targeting the AMPK pathway for the treatment of Type 2 diabetes. Front Biosci (Landmark Ed) 14: 3380-3400, 2009.

24. Xu Q and Si LY: Protective effects of AMP-activated protein kinase in the cardiovascular system. J Cell Mol Med 14: 2604-2613, 2010

25. Hawley SA, Ross FA, Chevtzoff C, Green KA, Evans A, Fogarty S, Towler MC, Brown LJ, Ogunbayo OA, Evans AM and Hardie DG: Use of cells expressing gamma subunit variants to identify diverse mechanisms of AMPK activation. Cell Metab 11: 554-565, 2010.

26. Bhatt MP, Lim YC, Kim YM and Ha KS: C-peptide activates AMPK $\alpha$ and prevents ROS-mediated mitochondrial fission and endothelial apoptosis in diabetes. Diabetes 62: 3851-3862, 2013.

27. Hawley SA, Pan DA, Mustard KJ, Ross L, Bain J, Edelman AM, Frenguelli BG and Hardie DG: Calmodulin-dependent protein kinase kinase-beta is an alternative upstream kinase for AMP-activated protein kinase. Cell Metab 2: 9-19, 2005.

28. Shaw RJ, Kosmatka M, Bardeesy N, Hurley RL, Witters LA, DePinho RA and Cantley LC: The tumor suppressor LKB1 kinase directly activates AMP-activated kinase and regulates apoptosis in response to energy stress. Proc Natl Acad Sci USA 101: 3329-3335, 2004.

29. Fisslthaler B and Fleming I: Activation and signaling by the AMP-activated protein kinase in endothelial cells. Circ Res 105: 114-127, 2009.

30. Liu L, Liu J, Wong WT, Tian XY, Lau CW, Wang YX, Xu G, $\mathrm{Pu} Y$, Zhu Z, Xu A, et al: Dipeptidyl peptidase 4 inhibitor sitagliptin protects endothelial function in hypertension through a glucagon-like peptide 1-dependent mechanism. Hypertension 60: 833-841, 2012. 